https://doi.org/10.15407/ujpe64.12.1125

Y. BESPALOV

Bogolyubov Institute for Theoretical Physics, Nat. Acad. of Sci. of Ukraine

(14b, Metrolohichna Str., Kyiv 03680, Ukraine; e-mail: yu.n.bespalov@gmail.com)

\title{
CATEGORIES: BETWEEN CUBES AND GLOBES. SKETCH I ${ }^{1}$
}

\begin{abstract}
For a finite partially ordered set I, we define an abstract polytope $\mathcal{P}_{I}$ which is a cube or a globe in the cases of discrete or linear poset, respectively. For a poset $P$, we have built a small category $\nabla_{P}$ with finite lower subsets in $P$ as objects. This category $\nabla_{P}=\diamond_{P}^{+} \diamond_{P}^{-}$is factorized into a product of two wide subcategories $\diamond_{P}^{+}$of faces and $\diamond_{P}^{-}$of degenerations. One can imagine a degeneration from $I$ to $J \subset I$ as a projection of an abstract polytope $\mathcal{P}_{I}$ to the subspace spanned by $J$. Morphisms in $\diamond_{P}^{+}$with fixed target $I$ are identified with faces of $\mathcal{P}_{I}$. The composition in $\diamond_{P}$ admits the natural geometric interpretation. On the category $\widehat{\nabla_{I}}$ of presheaves on $\nabla_{I}$, we construct a monad of free category in two steps: for a terminal presheaf, the free category is obtained via a generalized nerve construction; in the general case, the cells of a nerve are colored by elements of the initial presheaf. Strict P-fold categories are defined as algebras over this monad. All constructions are functorial in P. The usual theory of globular and cubical higher categories can be translated in a natural way into our general context.

Ke ywords: category theory.
\end{abstract}

\section{Introduction}

Usually, the higher-dimensional category means a category with globular cells $[1,5]$. Another version useful in many contexts is cubical categories [3]. Here, we introduce and study intermediate cases of higherdimensional categories with cells whose shapes are determined by partially ordered sets (posets). The concept of abtsract polytope ([6]) generalizes the notions of convex polytope, polyhedral fan, and tessellations and shows itself very useful in the regular context, when the symmetry group acts transitively on full flags. We define an abstract polytope $\mathcal{P}_{I}$ for each finite poset $I$. This is a cube or a globe in the cases of discrete or linear poset, respectively. In the general case, this abstract polytope is irregular, not a convex polytope or fan.

Each face of an abstract polytope $\mathcal{P}_{I}$ is again an abstract polytope $\mathcal{P}_{J}$ for some lower set $J \in \mathcal{O}(I)$, i.e., for each poset $P$, these data are organized into a category of faces $\diamond_{P}^{+}$. Then, for a poset $P$, we build a small category $\nabla_{P}$, where objects are identified with polytopes $\mathcal{P}_{I}$ for finite lower subsets $I \subseteq P$. Morphisms are uniquely factorized into a product of a degeneration from $\nabla_{P}^{-}:=\mathcal{O}(P)^{\text {op }}$ followed by a face from $\diamond_{P}^{+}$of faces.

(C) Y. BESPALOV, 2019

ISSN 2071-0194. Ukr. J. Phys. 2019. Vol. 64, No. 12
On the category $\widehat{\nabla_{P}}$ of presheaves on $\diamond_{P}$, we construct a monad of free category in two steps: for the terminal presheaf $1 \in \widehat{\widehat{\nabla}_{P}}$, the free category is obtained via a generalized nerve construction; for a general presheaf $X: \diamond_{P} \rightarrow \mathcal{S}$ et, to obtain $\mathcal{F} X$, cells of the nerve of 1 are colored by elements of the initial presheaf $X$. Strict $P$-fold categories are defined as algebras over this monad. The functoriality of all constructions in $P$ can be used to translate. The usual theory of globular and cubical higher categories in a natural way can bed into our general context.

\section{Posets and Abstract Polytopes}

Let $P$ be a partially ordered set (poset). A subset $I \subseteq P$ is called a lower set (resp., upper set), if, for each $x \in I$ and $y \in P$ with $y \leq x$ (resp., $y \geq x$ ), we have $y \in I$. Note that lower sets in $P$ are upper sets in the opposite poset $P^{\text {op }}$ and vice versa; $I \subseteq P$ is a lower set, if its complement $P \backslash I$ is an upper set.

For an arbitrary subset $X \subseteq P$, we denote, by $X^{\downarrow}$ (resp., $X^{\uparrow}$ ), the smallest lower set (resp., the greatest upper set) containing $X$. In the case of a singleton, the lower set $\{x\}^{\downarrow}$ is called principle.

\footnotetext{
1 This work is based on the results presented at the XI BolyaiGauss-Lobachevskii (BGL-2019) Conference: Non-Euclidean, Noncommutative Geometry and Quantum Physics.
} 
Further, we consider posets, where all principle lower sets are finite. Denote, by $\mathcal{O}(P)$, the set of finite lower sets in a such poset $P$. Unions of finite families and intersections of nonempty families of finite lower sets are again finite lower sets. So, $\mathcal{O}(P)$ is a distributive lattice with the smallest element $\emptyset$.

For a subset $X \subseteq P$ with induced order, we denote, by $\max (X)$ and $\min (X)$, the sets of maximal and minimal elements in $X$.

For two posets $P$ and $Q$, one can consider the product $P \times Q$, coproduct $P \sqcup Q$, and linear sum $P+Q$ which is a disjoint union, where the order relation $\leq_{P+Q}$ is the union of $\leq_{P}, \leq_{Q}$ and $P \times Q$.

Definition 1 ([6]). An abstract polytope of (finite) rank $n(n \geq-1)$ (or $n$-polytope) is a poset $\mathcal{P}$ with a strictly monotone rank function

$\operatorname{rank}_{\mathcal{P}}: \mathcal{P} \rightarrow\{-1<0<\ldots<n\}$.

Elements of

$\mathcal{P}_{i}=\{F \in \mathcal{P} \mid \operatorname{rank} F=i\}$

are called $i$-faces. The following 4 axioms are satisfied:

1. $\mathcal{P}_{-1}=\{\widehat{0}\}, \mathcal{P}_{n}=\{\widehat{1}\}$. (Hence, $\widehat{0}$ is the least face, and $\hat{1}$ is the greatest face.) Other faces are called proper. Denote

$\mathcal{P}^{>}=\mathcal{P} \backslash\{\widehat{0}\}, \quad \mathcal{P}^{<}=\mathcal{P} \backslash\{\widehat{1}\}, \quad \mathcal{P}^{<>}=\mathcal{P} \backslash\{\widehat{0}, \widehat{1}\}$.

2. Each flag (maximal chain) contains $n+2$ faces.

3. It is strongly connected, i.e., for every interval $\left[F_{1}, F_{2}\right]$ with $\operatorname{rank} F_{2}-\operatorname{rank} F_{1}>2$ and $F, F^{\prime} \in$ $\in\left(F_{1}, F_{2}\right)$, there is a way to pass from $F$ to $F^{\prime}$ via adjacent faces in $\left(F_{1}, F_{2}\right)$.

4. The diamond condition is satisfied: every 1section is a line segment, i.e., if $F_{1}$ and $F_{2}\left(F_{1}<F_{2}\right)$ differ in rank by 2 , then there are exactly two intermediate faces $F, F^{\prime}$ in the interval $\left(F_{1}, F_{2}\right)$.

Example 1. Faces of a convex polytope (including the empty set) ordered by inclusion form an abstract polytope.

The dual abstract polytope is defined as the opposite poset $\mathcal{P}^{\text {op }}$. This definition agrees with the (barycenric) duality for convex polytopes.

Proposition 1. Given a finite poset $I$, there is an abstract polytope $\mathcal{P}_{I}$, where an element of $\mathcal{P}_{I}^{>}$is the pair of a lower set $J \subseteq I$ and a function $f: \min (I \backslash$ $\backslash J) \rightarrow \mathbf{2}=\{0<1\}$ with $\operatorname{rank}(J, f)=|J|$. The order is $(K, g) \leq(J, f)$, iff $K \subseteq J$ and $f=g$ on $\min (I \backslash$ $J) \cap \min (I \backslash K)$.

Proposition 2. This correspondence extends to the functor $\mathcal{P}_{-}$from the category of finite posets and bijectons to the full subcategory of abstract polytopes in the category of posets. For two partial orders $\leq_{1} \subseteq \leq_{2}$ on the same finite set $I$ : a face $(J, f) \in$ $\in \mathcal{P}_{I, \leq_{1}}$ is mapped to a face of $\mathcal{P}_{I, \leq_{2}}$ presented by $I \backslash(I \backslash J)^{\uparrow} \leq_{2} \in \mathcal{O}\left(I, \leq_{2}\right)$ and the restriction of $f$ from $\min _{\leq_{1}}(I \backslash J)$ to $\min _{\leq_{2}}(I \backslash J)^{\uparrow} \leq_{2}$.

Remark 1. The dual polytope $\mathcal{P}_{I^{\mathrm{op}}}^{\mathrm{op}}$ admits a geometric realization in the linear space $\mathbb{R} I$ of formal sums $x=\sum_{i \in I} x_{i} \cdot i, x_{i} \in \mathbb{R}$. A subset $J \subseteq I$ determines a linear subspace $\mathbb{R} J \subseteq \mathbb{R} I$. Let us consider a configuration generated by the subspaces $\mathbb{R} J$ spanned on lower subsets $J \subseteq I$. The subspaces of codimension 1 split each given subspace into polyhedral cones. Note that, for an element $j \in J$ of a lower set, $J \backslash\{j\}$ is a lower set, iff $j \in \max J$. So, we obtain a collection of cones $C_{J}^{f}$ indexed by pairs $(J, f)$ of a lower subset $J \subseteq I$ and a function $f: \max J \rightarrow \mathbf{2}:=\{0,1\}$, where $C_{J}^{f}:=\left\{\left(x_{j}\right)_{j \in J} \in \mathbb{R} J \mid \forall j \in \max J \sum_{j}(-1)^{f(j)} x_{j} \leq 0\right\}$.

This is a poset ordered by inclusion: $C_{K}^{g} \subseteq C_{J}^{f}$ iff $K \subseteq J$ and $f=g$ on $\max J \cap \max K$.

Intersections of the above cones with the unit sphere $S_{I}:=\left\{x \mid \sum_{i \in I} x_{i}^{2}=1\right\}$ determine a spherical tessellation of $S_{I}$ by a fragment of big subspheres $S_{J}=$ $=\mathbb{R} J \cap S_{I}$. The greatest element is identified with the closed unit ball.

According to [4], one can define four types of products (join, sum, direct product, topological product) for a family $\left(\mathcal{P}_{j}\right)_{j \in J}$ of abstract polytopes taking a Cartesian product of undelying posets for $\mathcal{P}_{j}, \mathcal{P}_{j}^{>}$, $\mathcal{P}_{j}^{<}$, and $\mathcal{P}_{j}^{<>}$, respectively.

The construction $\mathcal{P}_{-}$turns the coproduct into a direct product and the ordered sum to an ordered sum:

Proposition 3. For finite posets $I_{1}$ and $I_{2}$,

$\mathcal{P}_{I_{1} \sqcup I_{2}}^{>} \cong \mathcal{P}_{I_{1}}^{>} \times \mathcal{P}_{I_{2}}^{>}, \quad \mathcal{P}_{I_{1}+I_{2}}^{<>} \cong \mathcal{P}_{I_{1}}^{<>}+\mathcal{P}_{I_{2}}^{<>}$.

Example 2. For a singleton $*, \mathcal{P}_{*}$ is an interval. If $I \cong \coprod_{I} *$ is a discrete poset, then $\mathcal{P}_{I}^{>} \cong \prod_{I} \mathcal{P}_{*}^{>}$ is a cube.

If $I \cong \sum_{I} *$ is totally ordered, then $\mathcal{P}_{I}^{<>} \cong$ $\cong \sum_{I} \mathcal{P}_{*}^{<>}$is a globe with a pair of proper faces in each dimension.

ISSN 2071-0194. Ukr. J. Phys. 2019. Vol. 64, No. 12 
A 2-element poset is either discrete or totally ordered. For 3-element poset, there exists three more possibilities:

$-\mathcal{P}_{* \sqcup(*+*)}^{>} \cong \mathcal{P}_{*}^{>} \times \mathcal{P}_{*+*}^{>}$is a cylinder;

$-\mathcal{P}_{(* \sqcup *)+*}^{<>} \cong \mathcal{P}_{* \sqcup *}^{<>}+\mathcal{P}_{*}^{<>}$is a "pillow";

$-\mathcal{P}_{*+(* \sqcup *)}^{<>} \cong \mathcal{P}_{*}^{<>}+\mathcal{P}_{* \sqcup *}^{<>}$is a "banana".

\section{Polytopical Categories}

A category $\mathcal{C}$ is factorized into a product of two subcategories $\mathcal{C}^{+}, \mathcal{C}^{-} \hookrightarrow \mathcal{C}$, if every morphism in $\mathcal{C}$ factors uniquely as a map in $\mathcal{C}^{-}$followed by a map in $\mathcal{C}^{+}$. Then we write $\mathcal{C}=\mathcal{C}^{+} \mathcal{C}^{-}$. (In this case, $\mathcal{C}^{+}$and $\mathcal{C}^{-}$are automatically wide subcategories.) So, the composition $f g$ in the opposite order with $f \in \mathcal{C}^{-}$, and $g \in \mathcal{C}^{+}$admits the unique factorization ${ }^{f} g \circ f^{g}$ with ${ }^{f} g \in \mathcal{C}^{+}$and $f^{g} \in \mathcal{C}^{-}$...

Entwining conditions:

- $\mathcal{C}^{+}$is a left module over $\mathcal{C}^{-}$:

${ }^{f_{1} f_{2}} g={ }^{f_{1}}\left({ }^{f_{2}} g\right), \quad{ }^{1} g=g$;

- $\mathcal{C}^{-}$is a right module over $\mathcal{C}^{+}$:

$f^{g_{1} g_{2}}=\left(f^{g_{1}}\right)^{g_{2}}, \quad f^{1}=f ;$

- "cocycle conditions":

$\left(f_{1} f_{2}\right)^{g}=f_{1}^{\left(f_{2} g\right)} \circ f_{2}^{g}, \quad 1^{g}=1 ;$

${ }^{f}\left(g_{1} g_{2}\right)={ }^{f} g_{1} \circ{ }^{\left(f^{g_{1}}\right)} g_{2}, \quad{ }^{f} 1=1$.

Theorem 1. Given a poset $P$, there exists a small category $\nabla_{P}=\diamond_{P}^{+} \diamond_{P}^{-}$factorized into a product of two wide subcategories $\diamond_{P}^{+}$of faces and $\diamond_{P}^{-}$of degenerations.

- The subcategory of degenerations $\diamond_{P}^{-}=$ $\mathcal{O}(P)^{\mathrm{op}}$. Denote, by $\iota_{I \subseteq J}$, the unique element of $\diamond_{P}^{-}(J, I)$ for $I \subseteq J$ in $\mathcal{O}(P)$.

- There exists a functor $\diamond_{P}^{+} \rightarrow \mathcal{O}(P)$ identical on objects. Equivalently, this means that if $\diamond_{P}^{+}(I, J)$ is non-empty, then $I \subseteq J$. In this case, $\diamond_{P}^{+}(I, J)$ is the set of functions $\llbracket I, J \rrbracket \rightarrow \mathbf{2}=\{0,1\}$, where

$\llbracket I, J \rrbracket=\{a \in J \backslash I \mid I \cup\{a\} \in \mathcal{O}(P)\}=\min (J \backslash I)$.

For $I \subseteq J \subseteq K$ in $\mathcal{O}(P)$, we have $\llbracket I, K \rrbracket \subseteq \llbracket I, J \rrbracket \sqcup$ $\sqcup \llbracket J, K \rrbracket$, and the composition of $f: \llbracket I, J \rrbracket \rightarrow \mathbf{2}$ and $g: \llbracket J, K \rrbracket \rightarrow \mathbf{2}$ is a function $g \circ f: \llbracket I, K \rrbracket \rightarrow \mathbf{2}$ with

$(g \circ f)(a)= \begin{cases}f(a), & \text { if } a \in \llbracket I, J \rrbracket, \\ g(a), & \text { if } a \in \llbracket J, K \rrbracket .\end{cases}$

ISSN 2071-0194. Ukr. J. Phys. 2019. Vol. 64, No. 12
This composition is associative with unit given by the unique function $\llbracket I, I \rrbracket=\emptyset \rightarrow \mathbf{2}$ for each $I \in \mathcal{O}(P)$.

- Entwining data: for $I \subseteq J \supseteq K$ in $\mathcal{O}(P)$ and $f: \llbracket I, J \rrbracket \rightarrow \mathbf{2}$,

$-\iota_{K \leq J}^{f}=\iota_{K \cap I \leq I}$

$-{ }^{\iota_{K} \leq J} f: \llbracket K \cap I, K \rrbracket \rightarrow \mathbf{2}$ is the restriction of $f$ on $\llbracket K \cap I, K \rrbracket \subseteq \llbracket I, K \rrbracket ;$

\section{P-Categories}

Denote, by $\widehat{\nabla_{P}}=\left[\nabla_{P}, \mathcal{S}\right.$ et $]$, the category of presheves $X: \diamond_{P} \rightarrow \mathcal{S}$ et. In particular, $\widehat{\diamond}_{*}$ is the category of oriented graphs with unital loops.

The $P$-category structure on a presheaf from $\widehat{\nabla_{P}}$ is given by a composition in each direction $a \in P$.

For $I \in \mathcal{O}(P)$ and $a \in I$, we consider a low set $I_{a}=I \backslash\{a\}^{\uparrow}$. For two different $a, b \in I$, the low sets $I_{a}$ and $I_{b}$ are different.

We have $\min \left(I \backslash I_{a}\right)=\{a\}$. So, we have a functor $\iota_{I, a}: \diamond_{*} \rightarrow \diamond_{P}$ whose image is a full subcategory with objects $I$ and $I_{a}$. For a presheaf $X \in \widehat{\nabla_{P}}$, the composition $X \circ \iota_{I, a} \in \widehat{\nabla_{*}}$.

For each $I \in \mathcal{O}(P)$ and discrete subposet $\{a \neq$ $\neq b\} \subseteq I$, we have a functor $\iota_{I, a, b}^{\prime}: \diamond_{* \sqcup *} \rightarrow \diamond_{P}$. For each $I \in \mathcal{O}(P)$ and ordered pair $\{a<b\} \subseteq I$, we have a functor $\iota_{I, a, b}^{\prime \prime}: \diamond_{*+*} \rightarrow \diamond_{P}$.

Definition 2. A strict P-category structure on a presheaf $X \in \widehat{\nabla_{P}}$ is given by a usual category structure on each oriented graph $X \circ \iota_{I, a}, a \in I \in \mathcal{O}(P)$ satisfying the compatibility conditions:

- For each $I \in \mathcal{O}(P)$ and discrete subposet $\{a \neq$ $\neq b\} \subseteq I$, the induced structure on the presheaf $X \circ \iota_{I, a, b}^{\prime}: \diamond_{* \sqcup *}^{\mathrm{op}} \rightarrow$ Set turns it into a strict double category.

- For each $I \in \mathcal{O}(P)$ and ordered pair $\{a<b\} \subseteq$ $\subseteq I$, the induced structure on the presheaf $X \circ \iota_{I, a, b}^{\prime \prime}$ : $\diamond_{*+*}^{\mathrm{op}} \rightarrow$ Set turns it into a strict 2-category.

Alternatively, we can describe a direct construction of the free $P$-category monad $\mathcal{F}$ on $\widehat{\nabla_{P}}$ together with a category $\Delta_{P}$ and a nerve analog.

Definition 3. Let $P$ be a partial order. A finite presheaf on $P$ is a functor $X: P^{\mathrm{op}} \rightarrow \mathcal{F}$ inSet to finite sets with finite support $\operatorname{supp} X:=\{p \in P \mid X(p) \neq$ $\neq \emptyset\}$. A partial order on a finite presheaf $X$ is a partial order on each $X(p)$ such that, for each ordered pair $p \leq q$ in $P$, the corresponding map $X(q) \rightarrow X(p)$ is monotone. The morphism of ordered presheaves on $P$ is a natural transformation $\varphi: X \rightarrow Y$ such that 
$\varphi(p): X(p) \rightarrow Y(p)$ is a monotone map for each $p \in P$. For a pair of partial orders on a presheaf $X$, we write $\leq \subseteq \leq^{\prime}$, if $x \leq y$ yields $x \leq^{\prime}$ y for each $p \in P$ and $x, y \in X(p)$. A total order on $X$ is the maximal element in the set of partial orders on $X$ with respect to the above inclusion $\subseteq$.

We denote, by $\Delta_{P}$, the skeletal category of totally ordered finite presheaves on a partial order $P$. The distributive lattice $\mathcal{O}(P)$ is identified with the full subcategory $[P] \hookrightarrow \Delta_{P}$ of presheaves $X$, where each $X(p)$ is the empty set or singleton 1 .

Example 3. If $P=1$ is the singleton, then $\Delta_{1}=$ $=\Delta$ is the category of finite ordinals $n:=\{0<1<$ $<\ldots<n-1\}$ and monotone maps.

For a finite product $\Delta_{P_{1} \times \ldots \times P_{n}}=\Delta_{P_{1}} \times \ldots \times \Delta_{P_{n}}$. In particular, $\Delta_{I}=\Delta^{I}$ for a finite discrete set $I$.

Let $P=1,2, \ldots, \omega$ be a locally finite ordinal. The total order on a finite presheaf $X \in \widehat{P}$ gets a linear order on each $X(p), p \in P$. Moreover, $\Delta_{n}$ is the category of functors $\left[n^{\mathrm{op}}, \Delta\right]$ or $n$-trees from [2].

For a presheaf $X: \mathcal{C}^{\text {op }} \rightarrow \mathcal{S}$ et, the corresponding discrete opfibration $\mathrm{el}(X)^{\text {op }} \rightarrow \mathcal{C}^{\text {op }}$ is the pullback of the universal opfibration $1 / \mathcal{S}$ et $\rightarrow \mathcal{S}$ et along the functor $X$; objects of $\mathrm{el}(X)$ are pairs $C \in \mathrm{Ob} \mathcal{C}$ and $x \in X(C)$, morphisms $(C, x) \rightarrow\left(C^{\prime}, x^{\prime}\right)$ are morphisms $f: C \rightarrow C^{\prime}$ in $\nabla_{P}$ such that $X(f)\left(x^{\prime}\right)=x$.

The totally ordered finite presheaf $X: P^{\text {op }} \rightarrow$ $\rightarrow \mathcal{F}$ inSet determines a presheaf $F_{X}: \diamond_{P}^{\text {op }} \rightarrow \mathcal{S}$ et, where $F_{X}(I)$ is the set of sections of the corresponding discrete opfibration $\mathrm{el}\left(\left.X\right|_{I}\right)^{\mathrm{op}} \rightarrow \diamond_{P}^{\mathrm{op}}$. The presheaf $F_{X}$ represent a complex of abstract polytopes glued together according to the total order. Elements of $\mathcal{F} 1$, the free $P$-category monad applied to the terminal presheaf $1 \in \widehat{\widehat{\nabla}_{P}}$, are identified with all such complexes. For $Y \in \widehat{\nabla_{P}}$, the elements of the free $P$-category $\mathcal{F} Y$ are elements of $\mathcal{F} 1$ colored by suitable elements of $Y$.

1. M.A. Batanin. Monoidal globular categories as natural environment for the theory of weak $n$-categories. Advances in Math. 136, 39 (1998).

2. M.A. Batanin. The Eckmann-Hilton argument, higher operads and $E_{n}$-spaces. Advances in Math. 217, 334 (2008).

3. R. Brown, P.J. Higgins, R. Sivera. Nonabelian Algebraic Topology. Filtered spaces, Crossed Complexes, Cubical Homotopy Groupoids. EMS Tracts in Math. 15, EMS (2011).

4. I. Gleason, I. Hubard. Products of abstract polytopes. J. Combinatorial Theory. Series A 157, 287 (2018).

5. T. Leinster. Higher Operads, Higher Categories (Cambridge University Press, 2003).

6. P. McMullen, E. Schulte. Abstract Regular Polytopes Encyclopedia of Mathematics and its Applications (Cambridge University Press, 2002), vol. 92.

Received 02.09.19

Ю. Беспалов

\section{КАТЕГОРІЇ: МІЖ КУБІЧНИМИ ТА СФЕРИЧНИМИ}

Р е $з$ ю м е

Вивчаються багатовимірні категорії, форма клітин яких залежить від частково-впорядкованої множини. 